\title{
Inguinal Hernia in Preterm Infants: Optimal Timing of He- rniorrhaphy to Prevent Preoperative Incarceration and Postoperative Apnea
}

\author{
Jaeho Shin, $\mathrm{MD}^{1}$ and Ga Won Jeon, $\mathrm{MD}, \mathrm{PhD}^{2}$ \\ ${ }^{1}$ Division of Pediatric Surgery, Department of Surgery, Inje University Busan Paik Hospital, Busan, Korea \\ ${ }^{2}$ Department of Pediatrics, Inje University Busan Paik Hospital, Inje University College of Medicine, Busan, Korea
}

\section{ABSTRACT}

Purpose: The objective of this study was to determine the optimal timing of inguinal herniorrhaphy in preterm infants to reduce the risks of preoperative incarceration and postoperative complications.

Methods: Preterm infants with gestational age (GA) of $<37$ weeks who had inguinal herniorrhaphy before the age of 6 months were enrolled. Early repair was defined as undergoing herniorrhaphy before discharge from the neonatal intensive care unit (NICU), and late repair was defined as undergoing herniorrhaphy as an outpatient after discharge from the NICU.

Results: The incidence rates of preoperative incarceration and recurrence were not significantly different between the two groups. Postoperative apnea and mechanical ventilation were more frequent in the early-repair group than in the late-repair group. Postoperative apnea was more frequent in the early-repair group after adjustments for GA and birth weight. However, no significant difference in postoperative mechanical ventilation was found between the two groups after adjustments for GA and birth weight. The incidence of postoperative apnea was associated with small weight at repair, early repair, general anesthesia, younger GA, small weight at birth, and bronchopulmonary dysplasia. Contralateral metachronous hernia was most frequent in infants with small weight at repair, early repair, very low birth weight (VLBW), male sex, and right-sided hernia.

Conclusion: Late repair was safe and did not increase the risk of incarceration or recurrence, but decreased the risks of postoperative apnea and metachronous hernia. Regional anesthesia could reduce the risk of postoperative apnea. Male infants born with VLBWs and right-sided hernia should be followed up carefully for metachronous hernia.

Key Words: Infant, premature; Hernia, inguinal; Herniorrhaphy; Infant, very low birth weight; Infant, newborn
Received: 10 May 2020

Revised: 22 June 2020

Accepted: 19 July 2020

Correspondence to: Ga Won Jeon, MD, $\mathrm{PhD}$

Department of Pediatrics, Inje Uni versity Busan Paik Hospital, Inje University College of Medicine, 75 Bokjiro, Busanjin-gu, Busan 47392, Korea Tel: +82-51-890-6497 Fax: +82-51-890-5830 E-mail: iamgawon@hanmail.net

Copyright(c)

By Korean Society of Neonatology. All right reserved.

This is an Open-Access article distributed under the terms of the Creative Commons At tribution Non-Commercial License (http:// creativecommons.org/licenses/by-nc/4.0), which permits unrestricted non-commercial use, distribution, and reproduction in any medium, provided the original work is properly cited. 


\section{INTRODUCTION}

Failure of the patent processus vaginalis to close results in indirect inguinal hernia ${ }^{1)}$. The incidence of inguinal hernia ranges from $3 \%$ to $5 \%$ in term infants but is significantly higher in pre term infants, approximately $10 \%$ to $30 \%^{2,3)}$. Though inguinal her niorrhaphy is one of the most common operations performed by pediatric surgeons, the optimal timing of herniorrhaphy in preterm infants admitted in neonatal intensive care units (NICUs) is still controversial.

The incidence of bowel incarceration while waiting for an operation for an inguinal hernia is especially high in preterm infants ${ }^{4)}$. Thus, herniorrhaphy shortly after diagnosis in the NICU is recommended to decrease the risks of incarceration and testicular ischemia $^{5,6)}$. Some researchers highly recommend herniorrhaphy as soon as possible in preterm or very low birth weight (VLBW) infants when diagnosis is confirmed because of their high risk for incarceration ${ }^{7}$. In fact, pediatric surgeons usually perform herniorrhaphy before NICU discharge to avoid the need for readmission for herniorrhaphy as well as to decrease the risk of incarceration. Infants discharged from an NICU with known inguinal hernia frequently visit the emergency department before herniorrhaphy because of concerns about the hernia, which can cause pressure to their families ${ }^{8)}$.

By contrast, researchers who advocate delayed operation are concerned about the increased risks of recurrence, postoperative apnea, and the need of mechanical ventilation associated with early operation before discharge from the NICU. Recent studies have found that herniorrhaphy before discharge from the NICU increased the risk of recurrence ${ }^{9)}$ and delayed operation after discharge from the NICU did not increase the risks for preoperative incarceration and recurrence ${ }^{10)}$. Early operation before NICU discharge did not prevent incarceration but prolonged the postoperative hospital stay and intubation as compared with operation after discharge from the NICU ${ }^{11)}$.

However, according to the survey of the American Academy of Pediatrics that detailed the timing of herniorrhaphy, up to $63 \%$ of surgeons perform herniorrhaphy before discharge from the $\mathrm{NICU}^{12}$. Some pediatric surgeons prefer herniorrhaphy shortly after diagnosis in the NICU, whereas others prefer herniorrhaphy just before discharge from the NICU, and still others prefer herniorrhaphy as an outpatient after discharge from the NICU, waiting until the proper postmenstrual age (PMA) and body weight to reduce anesthetic risks and technical challenges.
Thus, the objective of this study was to determine the optimal timing of inguinal herniorrhaphy in preterm infants in the NICU to reduce the risks of preoperative incarceration in consideration of the possibilities of anesthetic and postoperative complications. We also evaluated the risk factors of postoperative apnea and contralateral metachronous inguinal hernia, based on which we suggest proper interventions to reduce the risks of postoperative apnea and metachronous hernia.

\section{MATERIALS AND METHODS}

Preterm infants with gestational age (GA) of $<37$ weeks who had been admitted to the NICU of Inje University Busan Paik Hospital (between January 2011 and December 2019) and had received inguinal herniorrhaphy by a single pediatric surgeon before the age of 6 months were enrolled. Early repair was defined as undergoing herniorrhaphy before discharge from the NICU, and late repair was defined as undergoing herniorrhaphy as an outpatient after discharge from the NICU according to the onset time of inguinal hernia.

Clinical data were collected retrospectively from the patients' medical records. Demographic factors and outcomes associated with the timing of repair were compared between the earlyand late-repair groups. The demographic factors included GA, birth weight, sex, body weight at repair, postnatal age at repair (postnatal weeks), PMA at repair, and bronchopulmonary dys plasia (BPD). The outcomes included incarceration of inguinal hernia before repair, recurrence of inguinal hernia after repair, postoperative apnea, postoperative mechanical ventilation, and contralateral metachronous hernia. The risk factors of postope rative apnea were evaluated between the apnea and non-apnea groups. The risk factors of the development of contralateral metachronous hernia in unilateral hernia were evaluated.

BPD was defined as oxygen dependency or respiratory support at 36 weeks of PMA, with oxygen treatment for at least the first 28 days ${ }^{13)}$. This disorder was categorized into three groups according to severity as follows: mild, moderate, or severe BPD. Postoperative apnea was defined as cessation of breathing for $>15$ seconds and typically accompanied by desaturation and bradycardia related to the operation or anesthesia, which was not detected immediately before operation. Postoperative mechanical ventilation was defined as the need for mechanical ventilation sup port for $>24$ hours due to the operation or anesthesia. General 
anesthesia was used when regional anesthesia failed technically. Contralateral metachronous inguinal hernia was defined as a unilateral inguinal hernia at the first presentation with symptoms that develops into a contralateral hernia after surgical repair ${ }^{14)}$.

We performed a $t$-test for the continuous variables with normal distributions and homogeneous variance, and used the MannWhitney $U$-test for variables without normal distributions or homogeneous variance, such as GA. The chi-square test or Fisher's exact test was used for the nominal variables. To adjust for the influences of GA and birth weight, multivariate logistic regression was used to determine the risk factors. All statistical analyses were performed using SPSS version 25.0 (IBM Co., Armonk, NY, USA). Data are presented as mean \pm standard deviation. $P$ values of $<0.05$ were considered statistically significant.

\section{RESULTS}

Among the 148 preterm infants enrolled, 60 had herniorrhaphy before discharge from the NICU (early-repair group) and 88 had herniorrhaphy as an outpatient after discharge from the NICU (late-repair group). Male and female infants accounted for $65 \%$ and $35 \%$ of the patients, respectively. Right, left, and bilateral laterality was found in 48,69 , and 31 patients, respectively. In the male infants, right, left, and bilateral laterality was found in 41, 37, and 18 patients, respectively. In the female infants, right, left, and bilateral laterality was found in 7,32 , and 13 patients, respectively.

\section{Comparison of the early- and late-repair groups}

GA and birth weight were smaller in the early-repair group than in the late-repair group $(29.4 \pm 2.9$ weeks vs. $31.7 \pm 3.6$ weeks, $P<0.001$; and $1,109 \pm 352$ g vs. $1,613 \pm 585$ g, $P<0.001$, respectively). Body weight at repair was smaller in the early-repair group than in the late-repair group $(2,291 \pm 386 \mathrm{~g}$ vs. $3,925 \pm 1,159 \mathrm{~g}, P<0.001)$. Postnatal age and PMA at repair were younger in the early-repair group than in the late-repair group $(9.2 \pm 3.5$ weeks vs. $11.5 \pm 5.0$ weeks, $P=0.003$; and $39.2 \pm 2.2$ weeks vs. $43.6 \pm 4$. 4 weeks, $P<0.001$, respectively). BPD was more frequent in the early-repair group than in the late-repair group. However, no significant difference in BPD was found between the two groups after adjustments for GA and birth weight (19 [31.7\%] vs. 11 [12.6\%], $P=0.005$; adjusted $P=0.202$; odds ratio $[\mathrm{OR}], 2.193 ; 95 \%$ confidence interval $[\mathrm{CI}]$, 0.657 to 7.323 ). The incidence of moderate to severe BPD was not significantly different between the two groups (5 [8.5\%] vs.
2 [2.3\%], $P=0.087)$. The frequency of herniorrhaphy performed under general anesthesia was not significantly different between the two groups (10 [16.7\%] vs. 21 [23.9\%], $P=0.291)$.

The incidence of preoperative incarceration or recurrence was not significantly different between the two groups. Two patients in the late repair-group had incarcerated inguinal hernias. One patient visited the outpatient clinic as soon as the mother detected the inguinal mass, and inguinal herniorrhaphy was performed immediately after inguinal ultrasonography. The other patient visited the emergency department with severe inguinal swelling. Eight hours had passed after the inguinal mass was detected. Successful manual reduction under general anesthesia was performed, and inguinal herniorrhaphy was performed after 3 days. The bowel was intact and was not resected.

Postoperative apnea and mechanical ventilation were more frequent in the early-repair group than in the late-repair group (16 [26.7\%] vs. 5 [5.7\%], $P<0.001$; and 7 [11.7\%] vs. 3 [3.4\%], $P=$ 0.049, respectively). Postoperative apnea was more frequent in the early-repair group after adjustments for GA and birth weight (adjusted $P=0.008$; OR, 5.382; 95\% CI, 1.551 to 18.676). However, no significant difference in postoperative mechanical ventilation was found between the two groups after adjustments for GA and birth weight (adjusted $P=0.253$; OR, 2.414; 95\% CI, 0.532 to 10.959). Contralateral metachronous hernia was more frequent in the early repair group $(12 / 41[29.3 \%]$ vs. $4 / 76$ [5.3\%], $P<0.001)$ (Table 1).

\section{Risk factors of postoperative apnea}

Among the 148 preterm infants enrolled, 21 presented with postoperative apnea. GA and birth weight were smaller in the apnea group (27.2 \pm 2.8 weeks vs. $31.4 \pm 3.2$ weeks, $P<0.001$; and $1,000 \pm 470$ g vs. $1,477 \pm 546$ g, $P<0.001$, respectively). Body weight at repair was smaller in the apnea group $(2,661 \pm 830 \mathrm{~g}$ vs. $3,362 \pm$ $1,254 \mathrm{~g}, P=0.002)$. Postnatal age at repair was rather older in the apnea group ( $13.1 \pm 4.8$ weeks vs. $10.2 \pm 4$.4 weeks, $P=0.016)$, and PMA at repair was not significantly different between the apnea and non-apnea groups $(40.9 \pm 5.6$ weeks vs. $41.9 \pm 4.0$ weeks, $P=$ 0.423). BPD and moderate to severe BPD were more frequent in the apnea group (16 [80.0\%] vs. 14 [11.0\%], $P<0.001$; and 6 $[30.0 \%]$ vs. $1[0.8 \%], P<0.001$, respectively). They were still more frequent in the apnea group than in the non-apnea group after adjustments for GA and birth weight (BPD [adjusted $P=0.001$; OR, 16.187; 95\% CI, 3.121 to 83.951]; moderate to severe BPD [adjusted $P=0.004 ;$ OR, 40.264, 95\% CI, 3.295 to 491.934 ]). 
Table 1. Comparison of the Early- and Late-Repair Groups

\begin{tabular}{lccc}
\hline Variable & Early-repair group $(\mathrm{n}=60)$ & Late-repair group $(\mathrm{n}=88)$ & $P$-value \\
\hline Gestational age (wk) & $29.4 \pm 2.9$ & $31.7 \pm 3.6$ & $<0.001$ \\
Birth weight (g) & $1,109 \pm 352$ & $1,613 \pm 585$ & $<0.001$ \\
Male sex & $40(66.7)$ & $56(63.6)$ & 0.705 \\
Body weight at repair (g) & $2,291 \pm 386$ & $3,925 \pm 1,159$ & $<0.001$ \\
Age at repair (wk) & $9.2 \pm 3.5$ & $11.5 \pm 5.0$ & 0.003 \\
PMA at repair (wk) & $39.2 \pm 2.2$ & $43.6 \pm 4.4$ & $<0.001$ \\
BPD & $19(31.7)$ & $11(12.6)$ & $0.005\left(0.202^{*}\right)$ \\
BPD (moderate to severe) & $5(8.5)$ & $2(2.3)$ & 0.087 \\
General anesthesia & $10(16.7)$ & $21(23.9)$ & 0.291 \\
Pre-operative incarceration & 0 & $2(2.3)$ & 0.240 \\
Recurrence & 0 & 0 & $>0.999$ \\
Post-operative apnea & $16(26.7)$ & $5(5.7)$ & $<0.001\left(0.008^{\dagger}\right)$ \\
Post-operative ventilator & $7(11.7)$ & $3(3.4)$ & $0.049\left(0.253^{\dagger}\right)$ \\
Laterality (right/left/bilateral) & $18 / 23 / 19$ & $30 / 46 / 12$ & 0.068 \\
Metachronous hernia & $12 / 41(29.3)$ & $4 / 76(5.3)$ & $<0.001$
\end{tabular}

Values are expressed as mean \pm standard deviation or number (\%).

*Adjusted $P$ for gestational age and birth weight $=0.202$ (OR, 2.193; 95\% CI, 0.657 to 7.323 ); ${ }^{\dagger}$ Adjusted $P$ for gestational age and birth weight $=0.008$ (OR, 5.382; 95\% CI, 1.551 to 18.676 ); ${ }^{*}$ Adjusted $P$ for gestational age and birth weight $=0.253$ (OR, 2.414; 95\% CI, 0.532 to 10.959 ).

Abbreviations: PMA, postmenstrual age; BPD, bronchopulmonary dysplasia; OR, odds ratio; CI, confidence interval.

Table 2. Risk Factors of Postoperative Apnea

\begin{tabular}{lccc}
\hline Variable & Apnea $(\mathrm{n}=21)$ & No-apnea $(\mathrm{n}=127)$ & $P$-value \\
\hline Gestational age (wk) & $27.2 \pm 2.8$ & $31.4 \pm 3.2$ & $<0.001$ \\
Birth weight (g) & $1,000 \pm 470$ & $1,477 \pm 546$ & $<0.001$ \\
Body weight at repair (g) & $2,661 \pm 830$ & $3,362 \pm 1,254$ & 0.002 \\
Age at repair (wk) & $13.1 \pm 4.8$ & $10.2 \pm 4.4$ & 0.016 \\
PMA at repair (wk) & $40.9 \pm 5.6$ & $41.9 \pm 4.0$ & 0.423 \\
BPD & $16(80.0)$ & $14(11.0)$ & $<0.001\left(0.001^{*}\right)$ \\
BPD (moderate to severe) & $6(30.0)$ & $1(0.8)$ & $<0.001\left(0.004^{\dagger}\right)$ \\
General anesthesia & $12(57.1)$ & $19(15.0)$ & $<0.001\left(<0.001^{\dagger}\right)$ \\
Early repair & $16(76.2)$ & $44(34.6)$ & $<0.001\left(0.030^{\S}\right)$
\end{tabular}

Values are expressed as mean \pm standard deviation or number (\%).

*Adjusted $P$ for gestational age and birth weight=0.001 (OR, 16.187; 95\% CI, 3.121 to 83.951); ${ }^{\dagger}$ Adjusted $P$ for gestational age and birth weight=0.004 (OR, 40.264; 95\% CI, 3.295 to 491.934 ); ${ }^{*}$ Adjusted $P$ for gestational age and birth weight $<0.001$ (OR, 8.116; $95 \%$ CI, 2.503 to 26.318 ); ${ }^{\circledR}$ Adjusted $P$ for gestational age and birth weight $=0.030$ (OR, 4.032; 95\% CI, 1.147 to 14.168).

Abbreviations: PMA, postmenstrual age; BPD, bronchopulmonary dysplasia; OR, odds ratio; CI, confidence interval.

Infants who underwent early repair were more frequent in the apnea group (16/21 [76.2\%] vs. 44/127 [34.6\%], $P<0.001$; adjusted $P$ for GA and birth weight=0.030; OR, 4.032; 95\% CI, 1.147 to 14.168). Herniorrhaphy performed under general anesthesia was more frequent in the apnea group (12/21 [57.1\%] vs. 19/127 [15.0\%], $P<0.001$; adjusted $P$ for GA and birth weight $<0.001$; OR, 8.116; 95\% CI, 2.503 to 26.318) (Table 2).

\section{Factors related to the development of metachronous hernia in unilateral hernia}

Sixteen preterm infants developed metachronous hernia (16/ 117 unilateral hernias, $13.7 \%$ ). Most of the metachronous hernias occurred in the male infants (15/16, 93.8\%). Body weight at repair was smaller in the metachronous hernia group than in the non-metachronous hernia group $(2,458 \pm 635$ g vs. 3,488 $\pm 1,247 \mathrm{~g}$, $P=0.002)$. Postnatal age and PMA at repair were younger in the metachronous hernia group ( $8.6 \pm 3.3$ weeks vs. $10.7 \pm 4.5$ weeks, 
Table 3. Factors Related to the Development of Metachronous Hernia in Unilateral Hernia

\begin{tabular}{|c|c|c|c|}
\hline Variable & $\begin{array}{l}\text { Metachronous } \\
\text { hernia }(n=16)\end{array}$ & $\begin{array}{c}\text { No } \\
\text { metachronous } \\
\text { hernia }(n=101)\end{array}$ & $P$-value \\
\hline Gestational age (wk) & $29.6 \pm 3.4$ & $30.9 \pm 3.6$ & 0.153 \\
\hline Birth weight (g) & $1,211 \pm 497$ & $1,468 \pm 582$ & 0.074 \\
\hline Male sex & $15(93.8)$ & $63(62.4)$ & 0.013 \\
\hline Body weight at repair (g) & $2,458 \pm 635$ & $3,488 \pm 1,247$ & 0.002 \\
\hline Age at repair (wk) & $8.6 \pm 3.3$ & $10.7 \pm 4.5$ & 0.032 \\
\hline PMA at repair (wk) & $38.6 \pm 2.2$ & $42.1 \pm 4.0$ & 0.001 \\
\hline Early repair & $12(75.0)$ & $29(28.7)$ & $<0.001$ \\
\hline
\end{tabular}

Values are expressed as mean \pm standard deviation or number (\%). Abbreviation: PMA, postmenstrual age.

$P=0.032$; and $38.6 \pm 2.2$ weeks vs. $42.1 \pm 4.0$ weeks, $P=0.001$, respectively). Of the 16 metachronous hernias, 11 (68.8\%) occurred in VLBW infants, and 12 (75\%) and four (25\%) were primary rightand left-sided hernias, respectively. Infants who underwent early repair were more frequent in the metachronous than in the nonmetachronous hernia group (12/16 [75.0\%] vs. 29/101 [28.7\%], $P<0.001$ ) (Table 3).

\section{DISCUSSION}

In the present study, early repair with herniorrhaphy before discharge from the NICU was performed at 9 weeks after birth and a PMA of 39 weeks, with a mean body weight of $2.3 \mathrm{~kg}$. Late repair with herniorrhaphy as an outpatient after discharge from the NICU was performed at 12 weeks after birth and a PMA of 44 weeks, with a mean body weight of $3.9 \mathrm{~kg}$.

Many pediatric surgeons perform herniorrhaphy before discharge from the NICU ${ }^{12)}$ to avoid readmission of the patient for herniorrhaphy or the risk of preoperative incarceration while waiting for operation. Herniorrhaphy before discharge from the NICU was associated with recurrence, prolonged hospital stay, and postoperative mechanical ventilation, whereas herniorrhaphy after discharge from the NICU was associated with incarceration $^{15)}$. The frequency of early repair did not increase between 1999 and 2011 according to Sulkowski et al. ${ }^{15)}$ but increased from $20 \%$ in 1998 to $45 \%$ in 2012 in another study ${ }^{16)}$. Institutional practices for inguinal herniorrhaphy seem to vary.

Although the incidence of incarceration differs among the studies, the overall reported incidence of incarceration ranges from $12 \%$ to $17 \%{ }^{17}$. The risk of bowel incarceration is especially high in preterm infants than in term infants ${ }^{18)}$. The incidence rates of incarceration were $25 \%$ and $13 \%$ at presentation and $11 \%$ and $3.4 \%$ at surgery in preterm and term infants, respectively, in the study of Hughes et al. ${ }^{18)}$. The incidence of incarceration was $>50 \%$ in the preterm infants, and emergency operations due to incarceration were more frequent in VLBW infants according to de Goede et al. ${ }^{19)}$. Thus, they recommended herniorrhaphy during NICU admission, especially in VLBW infants, who have a high risk of incarceration while waiting for operation. By contrast, the incidence of incarceration was not different between preterm and term infants, and was low in both groups according to a nationwide survey in Taiwan $(0.43 \%$ in preterm infants vs. 0.41 $\%$ in term infants, $P=0.959)^{20)}$. Relatively few incarcerations were reported in the present study. Only two cases of incarceration occurred before repair in the late-repair group, and no incarceration occurred in the early-repair group in the present study. Late repair with herniorrhaphy after discharge from the NICU did not increase the risk of incarceration in the present study. The result is similar to the finding that the waiting time for re pair of a reducible hernia is not related to the increased risk of incarceration ${ }^{21)}$. Olesen et al. ${ }^{22)}$ reported that herniorrhaphy does not need to be delayed unnecessarily because of the increased risk of incarceration. However, delayed repair can be justified in certain cases such as those with increased risk of anesthesia complication $^{22)}$.

Early repair with herniorrhaphy before discharge from the NICU was associated with increased risk of recurrence ${ }^{9)}$. The overall incidence of recurrence after herniorrhaphy is less than $1 \%{ }^{17)}$. However, the reported incidence of recurrence ranges from $1 \%$ to $9 \%$. Half of the recurrent cases occurred within 1 year after herniorrhaphy ${ }^{18)}$. No recurrent hernias were reported in the present study. The patients underwent routine follow-up as outpatients at the department of pediatric surgery a week after herniorrhaphy. No more routine follow-up visits were made if no complications occurred. This might have led to an underestimation of the recurrence rate.

Postoperative apnea was associated with low GA at birth and PMA at the time of operation ${ }^{8)}$. In the present study, postoperative apnea was more common in the early-repair group than in the late-repair group. GA and birth weight were lower in the apnea group as expected; however, PMA at repair was not lower in the apnea group. PMA was around 41 weeks in both groups. Body weight at repair was smaller in the apnea group. We suggest that body weight at repair is also or even more important than PMA 
in terms of the occurrence of postoperative apnea. According to the survey of the American Academy of Pediatrics, $10 \%$ of surgeons wait until infants reach a PMA of 50 weeks and $3 \mathrm{~kg}$ of body weight, which implies that surgeons also consider the importance of both body weight and PMA at repair ${ }^{12)}$. BPD and moderate to severe BPD were risk factors of postoperative apnea in the present study and were still significant factors for the development of postoperative apnea after adjustments for GA and birth weight. General anesthesia was also a risk factor of postoperative apnea in the present study and was more frequent in the apnea group. Regional anesthesia without preoperative sedatives reduced the risk of postoperative apnea, and regional anesthesia in inguinal herniorrhaphy was safer than general anesthesia according to a meta-analysis ${ }^{23}$. General anesthesia was associated with prolonged anesthesia time and increased risk of postoperative intubation as compared with regional anesthesia ${ }^{24)}$. Thirteen percent of the patients in the early-repair group required postoperative intubation over 48 hours according to Lee et al. ${ }^{11)}$. Similarly, we found that $12 \%$ of the patients in the early-repair group required postoperative ventilation support in the present study ( $12 \%$ in the early-repair group vs. $3 \%$ in the laterepair group).

The overall incidence of contralateral metachronous hernia ranged from $6 \%$ to $10 \%$ of all cases of unilateral hernia, reaching up to $12.3 \%$ according to Lee et al. ${ }^{14)}$. This correlates with the incidence rate of $13.7 \%$ in the present study. Primary left-sided hernia, younger age, and prematurity increase the risk of contralateral metachronous hernia ${ }^{25}$. Metachronous hernia was more frequent in VLBW infants, with an incidence of $10 \%$ of all cases of unilateral hernia, which was more frequent in primary rightsided hernias (primary right-sided hernia: primary left-sided hernia ratio, 9:4) and males ${ }^{7)}$. This correlates with our results. Metachronous hernia was more common in VLBW infants (11/ 16 metachronous hernias), primary right-sided hernia (primary right-sided hernia: primary left-sided hernia ratio, 12:4), and males (male:female ratio, 15:1) in the present study. We recommend that VLBW infants with primary right-sided hernia should be followed up carefully because of their risk of developing metachronous hernia. Demouron et al. ${ }^{26)}$ reported that in female preterm infants, metachronous hernia was more common in those with primary right-sided hernias, perhaps because right-sided inguinal hernias are more common than left-sided inguinal hernias. Metachronous hernia was more frequent in primary left-sided hernias in another study $^{27)}$. By contrast, no significant difference in laterality was found in another study ${ }^{10)}$. Thus, the laterality of metachronous hernia is still controversial. Metachronous hernia was more frequent in the early-repair group in the present study. Moreover, smaller body weight at repair, younger age, and younger PMA at repair were associated with increased risk of metachronous hernia.

Inguinal hernia is twice as common on the right side than on the left side in term infants. However, bilateral inguinal hernia is more frequent in preterm infants than in term infants ${ }^{28)}$. The incidence rate of bilateral presentation was $21 \%$, and that of rightsided presentation was relatively low (32\%) in the present study, perhaps because all the enrolled infants were preterm. Male predominance was observed in the term infants, with a male-tofemale ratio of 5:1 but was not significant in the preterm infants ${ }^{17)}$. The male-to-female ratio was 1.8:1 in the present study.

This study has limitations. Assignment to the early- and laterepair groups was not randomized because of retrospective nature of the study. The subjects were allocated according to the onset time of inguinal hernia (before or after discharge from the NICU). Thus, confounding factors might exist despite the adjustments for GA and birth weight because of the retrospective nature of this study.

In summary, late repair was safe and did not increase the risk of incarceration or recurrence, and instead, decreased the risk of postoperative apnea and contralateral metachronous hernias in preterm infants with inguinal hernia. Postoperative apnea was associated with small body weight at repair, early repair, general anesthesia, younger GA, small body weight at birth, and BPD, as expected. We suggest that greater body weight at repair and regional anesthesia could reduce the risk of postoperative apnea. Male infants born with VLBW and a primary right-sided hernia are likely to develop a contralateral metachronous hernia. Thus, late repair can be considered to decrease the risk of contralateral metachronous hernia in such patients, who should be followed up carefully in case they develop a contralateral metachronous hernia. Further randomized prospective studies to compare the timing of herniorrhaphy are required.

\section{ARTICLE INFORMATION}

\section{Ethical statement}

This study was reviewed and approved by the Institutional Review Board of Inje University Busan Paik Hospital (identifica- 
tion code: 20-0032) in accordance with the Declaration of Helsinki. The Institutional Review Board waived the need for informed consent for this retrospective chart review.

\section{Conflicts of interest}

No potential conflict of interest relevant to this article was reported.

\section{Author contributions}

Conception or design: J.S., G.W.J.

Acquisition, analysis, or interpretation of data: J.S, G.W.J.

Drafting the work or revising: J.S, G.W.J.

Final approval of the manuscript: J.S, G.W.J.

\section{ORCID}

Jaeho Shin https://orcid.org/0000-0002-5393-542X

GaWon Jeon https://orcid.org/0000-0002-8206-9727

\section{Acknowledgments}

None

\section{REFERENCES}

1. Pini Prato A, Casaccia G, Arnoldi R. Timing and management of inguinal hernia in the premature baby. Eur J Pediatr Surg 2017;27:472-7.

2. Khan FA, Zeidan N, Larson SD, Taylor JA, Islam S. Inguinal hernias in premature neonates: exploring optimal timing for repair. Pediatr Surg Int 2018;34:1157-61.

3. Kumar VH, Clive J, Rosenkrantz TS, Bourque MD, Hussain N. Inguinal hernia in preterm infants ( $<$ or $=32$-week gestation). Pediatr Surg Int 2002;18:147-52.

4. Steven M, Greene O, Nelson A, Brindley N. Contralateral inguinal exploration in premature neonates: is it necessary? Pediatr Surg Int 2010;26:703-6.

5. Vaos G, Gardikis S, Kambouri K, Sigalas I, Kourakis G, Petoussis G. Optimal timing for repair of an inguinal hernia in premature infants. Pediatr Surg Int 2010;26:379-85.

6. Gholoum S, Baird R, Laberge JM, Puligandla PS. Incarceration rates in pediatric inguinal hernia: do not trust the coding. J Pediatr Surg 2010;45:1007-11.

7. Pini Prato A, Rossi V, Mosconi M, Disma N, Mameli L, Montobbio $\mathrm{G}$, et al. Inguinal hernia in neonates and ex-preterm: complications, timing and need for routine contralateral ex ploration. Pediatr Surg Int 2015;31:131-6.

8. Duggan EM, Patel VP, Blakely ML. Inguinal hernia repair in pre mature infants: more questions than answers. Arch Dis Child Fetal Neonatal Ed 2015;100:F286-8.

9. Masoudian P, Sullivan KJ, Mohamed H, Nasr A. Optimal timing for inguinal hernia repair in premature infants: a systematic review and meta-analysis. J Pediatr Surg 2019;54:1539-45.

10. Youn JK, Kim HY, Huh YJ, Han JW, Kim SH, Oh C, et al. Inguinal hernia in preterms in neonatal intensive care units: optimal timing of herniorrhaphy and necessity of contralateral exploration in unilateral presentation. J Pediatr Surg 2018;53: 2155-9.

11. Lee SL, Gleason JM, Sydorak RM. A critical review of premature infants with inguinal hernias: optimal timing of repair, incar ceration risk, and postoperative apnea. J Pediatr Surg 2011;46: 217-20.

12. Antonoff MB, Kreykes NS, Saltzman DA, Acton RD. American Academy of Pediatrics section on surgery hernia survey re visited. J Pediatr Surg 2005;40:1009-14.

13. Kinsella JP, Greenough A, Abman SH. Bronchopulmonary dysplasia. Lancet 2006;367:1421-31.

14. Lee $\mathrm{CH}$, Chen Y, Cheng CF, Yao CL, Wu JC, Yin WY, et al. Incidence of and risk factors for pediatric metachronous contralateral inguinal hernia: analysis of a 17-year nationwide database in Taiwan. PLoS One 2016;11:e0163278.

15. Sulkowski JP, Cooper JN, Duggan EM, Balci O, Anandalwar SP, Blakely ML, et al. Does timing of neonatal inguinal hernia repair affect outcomes? J Pediatr Surg 2015;50:171-6.

16. Gulack BC, Greenberg R, Clark RH, Miranda ML, Blakely ML, Rice HE, et al. A multi-institution analysis of predictors of timing of inguinal hernia repair among premature infants. J Pediatr Surg 2018;53:784-8.

17. Ein SH, Njere I, Ein A. Six thousand three hundred sixty-one pediatric inguinal hernias: a 35-year review. J Pediatr Surg 2006; 41:980-6.

18. Hughes K, Horwood JF, Clements C, Leyland D, Corbett HJ. Complications of inguinal herniotomy are comparable in term and premature infants. Hernia 2016;20:565-9.

19. de Goede B, Verhelst J, van Kempen BJ, Baartmans MG, Langeveld HR, Halm JA, et al. Very low birth weight is an in dependent risk factor for emergency surgery in premature infants with inguinal hernia. J Am Coll Surg 2015;220:347-52.

20. Fu YW, Pan ML, Hsu YJ, Chin TW. A nationwide survey of incidence rates and risk factors of inguinal hernia in preterm children. Pediatr Surg Int 2018;34:91-5.

21. Chang SJ, Chen JY, Hsu CK, Chuang FC, Yang SS. The incidence of inguinal hernia and associated risk factors of incarceration in pediatric inguinal hernia: a nation-wide longitudinal population-based study. Hernia 2016;20:559-63.

22. Olesen CS, Mortensen LQ, Oberg S, Rosenberg J. Risk of incarceration in children with inguinal hernia: a systematic review. Hernia 2019;23:245-54. 
23. Jones LJ, Craven PD, Lakkundi A, Foster JP, Badawi N. Regional (spinal, epidural, caudal) versus general anaesthesia in preterm infants undergoing inguinal herniorrhaphy in early infancy. Cochrane Database Syst Rev 2015;2015:CD003669.

24. Gurria J, Kuo P, Kao A, Christensen L, Holterman A. General endotracheal vs. non-endotracheal regional anesthesia for elective inguinal hernia surgery in very preterm neonates: a single institution experience. J Pediatr Surg 2017;52:56-9.

25. Fraser JD, Snyder CL. Inguinal hernias and hydroceles. In: Holcomb GW, Murphy JP, Ostlie DJ, editors. Ashcraft's pediatric surgery. 6th ed. London: Saunders/Elsevier, 2014:679-88.
26. Demouron M, Delforge X, Buisson P, Hamzy M, Klein C, Haraux E. Is contralateral inguinal exploration necessary in preterm girls undergoing inguinal hernia repair during the first months of life? Pediatr Surg Int 2018;34:1151-5.

27. Zamakhshardy M, Ein A, Ein SH, Wales PW. Predictors of metachronous inguinal hernias in children. Pediatr Surg Int 2009;25:69-71.

28. Burgmeier C, Dreyhaupt J, Schier F. Comparison of inguinal hernia and asymptomatic patent processus vaginalis in term and preterm infants. J Pediatr Surg 2014;49:1416-8. 